\title{
Study on Multi-Knapsack Problem Based on Improved Artificial Fish School Algorithm
}

\author{
Lei Qin ${ }^{1,2, a}$, Kang Zhou ${ }^{1, b}$ \\ ${ }^{1}$ School of mathematics and computer, Wuhan Polytechnic University, Wuhan 430023, China; \\ ${ }^{2}$ School of Automation, Huazhong University of Science and Technology, Wuhan 430074, China \\ aqinlei@whpu.edu.cn, b'zhoukang1965@163.com
}

Keywords: Multi-knapsack problem; Artificial Fish School Algorithm; coding.

\begin{abstract}
Multi-knapsack problem (MKP) is one of the typical NP problem in optimization field, because the computing complexities of the traditional algorithms are high or they have slow convergence speeds, this paper proposes an improved artificial fish school algorithm (IAFSA) for MKP. Firstly, the integer coding was used in the input ways of knapsacks, secondly, IAFSA adopted the strategy of "random repair" to repair infeasible artificial fish coding and inadequate artificial fish coding, and the coding after taking behaviors were improved and repaired, finally, IAFSA was verified by an example. Experimental results show that the advantage of IAFSA over other AFSA algorithms mainly lies in its faster convergence speed, stronger robustness, and its convergence to the optimal solution with greater probability.
\end{abstract}

\section{Introduction}

Multi-knapsack problem (MKP) is a NP problem in the field of combinatorial optimization, MKP refers to when the sum of the goods weights in each knapsack shall not exceed the capacity of the knapsack, it achieves the maximum of the total values of all goods. There are many ways for MKP, such as exact algorithm, genetic algorithm, the discrete particle swarm optimization, ant colony algorithm, etc [1-5]. These algorithms often have slow convergence speeds or their computational complexities are high, In order to faster and more efficiently find the optimal solution of MKP, this paper tries to apply the artificial fish school algorithm (AFSA) in MKP. AFSA is a new bottom-up optimization strategy [6-8], researchers have applied AFSA and its extension algorithms in some combinatorial optimization problems such as regional economic system optimization, Vehicle Routing Problem and Travelling Salesman Problem.

Now that AFSA has succeed in combinatorial optimization problems, according to the characteristics of MKP, the paper designs an improved AFSA (IAFSA) for MKP. IAFSA introduces the "random repair" strategy, during the initialization and iteration of AFSA the strategy repairs infeasible artificial fish coding and inadequate artificial fish coding from beginning to end, in addition, greedy algorithm and value density sorting strategy have also been used to AFSA in order to verify the validity of IAFSA for MKP.

\section{The mathematical model of MKP}

Given $n$ goods, the weights of the goods are $w_{1}, w_{2}, \cdots, w_{n}$, their values of $p_{1}, p_{2}, \cdots, p_{n}$ respectively. Given $m$ knapsacks, the capacities of the knapsacks are $b_{1}, b_{2}, \cdots, b_{m}$ respectively. Demands finding $m$ mutually disjoint subsets of $n$ goods, they corresponding be loaded into $m$ knapsacks, the sum of the values of all loading goods should be the maximum, the mathematical model is described as follows.

$$
\begin{aligned}
& \max Z=\sum_{j=1}^{m} \sum_{i=1}^{n} p_{i} x_{i j} \\
& \text { s.t. } \quad \sum_{j=1}^{m} x_{i j}=0 \text { or } 1, \quad i=1,2, \cdots, n
\end{aligned}
$$




$$
\begin{aligned}
& \sum_{i=1}^{n} w_{i} x_{i j} \leq b_{j}, j=1,2, \cdots, m \\
& x_{i j}=0 \text { or } 1, \quad i=1,2, \cdots, n, j=1,2, \cdots, m
\end{aligned}
$$

\section{AFSA}

The basic definitions. The state of the artificial fish individual can be expressed as a vector $X=\left(x_{1}, x_{2}, \cdots, x_{n}\right)$, in which $x_{i}(i=1,2, \cdots, n)$ are the optimization variables, the food concentration of an artificial fish in the current location is represented as $Y=f(X)$, in which $Y$ is the objective function value, the distance between artificial fish individuals $X_{i}$ and $X_{j}$ is expressed as $d_{i j}=\left\|X_{i}-X_{j}\right\|, N$ is the size of the artificial fish school, visual is defined as the perception distance of an artificial fish vision, step is the biggest step length of an artificial fish moving, $\delta$ is the crowding factor, $0<\delta<1$, and Try number is the biggest probe number of an artificial fish for food.

The descriptions and choices of the behaviors. AFSA mainly consists of several behaviors, such as foraging, clustering, tailgating, etc, moreover the bulletin board is used to record the state of the optimal artificial fish individual. When each individual is in the process of optimization, the artificial fish is testing its own state and the state of the bulletin board after each action, if his state is superior to the state of the bulletin board, the latter will be updated, and make the bulletin board record the optimal state of the artificial fish school.

According to the nature of the MKP, make a judgment about the current environment of the artificial fish, so as to select the most optimal behavior and execute it.

The process of AFSA. The process of AFSA is shown in Fig.1.

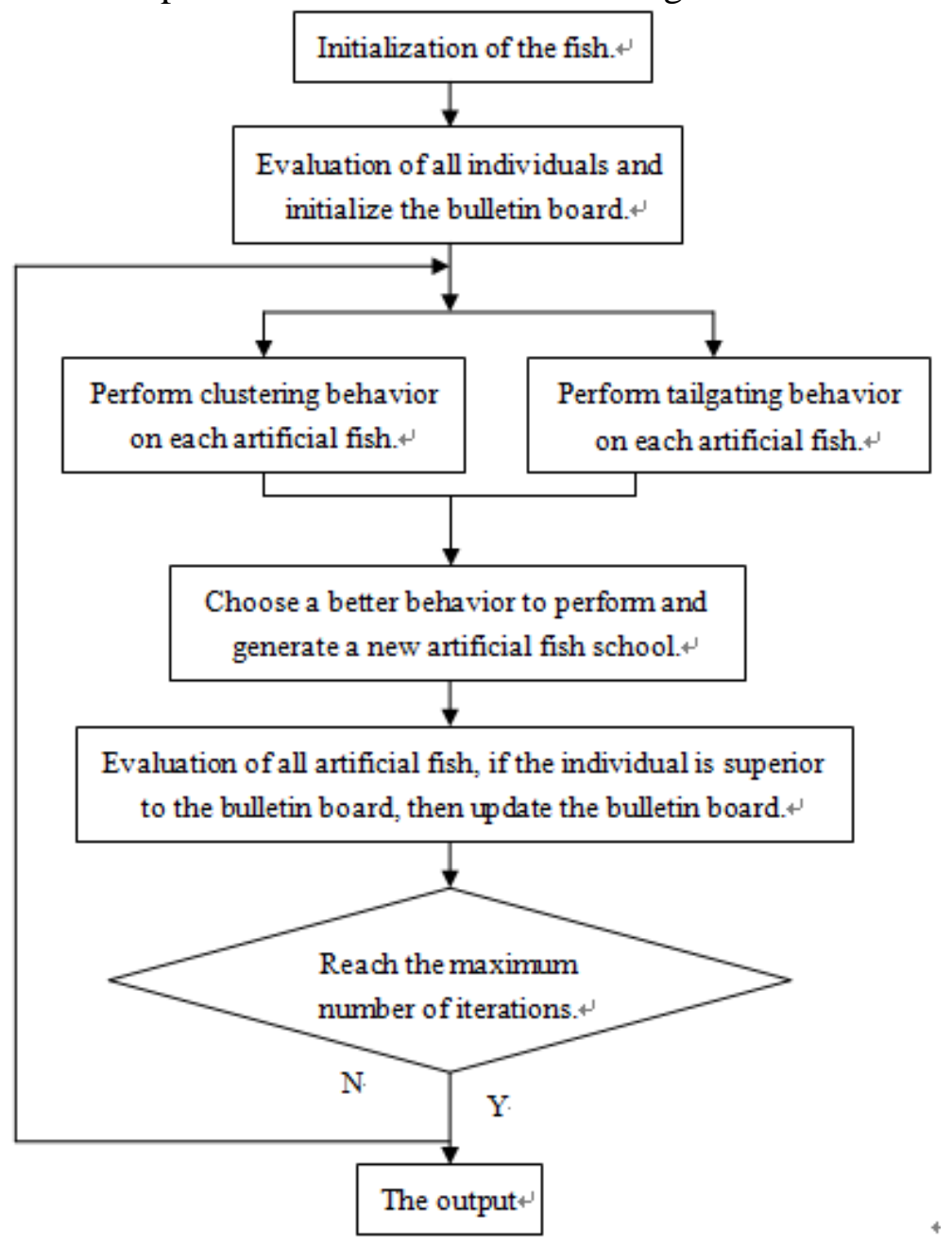

Fig.1 AFSA flow chart 


\section{IAFSA for MKP}

Artificial fish coding and decoding method. In this paper, an $1 \times n$ integer coding is used to construct an artificial fish individual, namely $a_{i}=\left[a_{i 1}, a_{i 2}, \cdots, a_{i n}\right]$, in which $a_{i j}(j=1,2, \cdots, n)$ is an integer in the interval $[0, m]$, i.e.

$$
a_{i j}=\left\{\begin{array}{cc}
k & \text { goods } j \text { is in knapsack } k,(k=1,2, \cdots, m) \\
0 & \text { otherwise }
\end{array}\right.
$$

Infeasible, inadequate artificial fish coding's repair. An infeasible artificial fish coding is defined as an artificial fish coding that doesn't meet the constraint conditions of MKP after decoding, i.e. equation (3), this paper chooses the most simple and effective way, namely "random repair" strategy. Random repair infeasible artificial fish coding is to take aim at all knapsacks that don't satisfy the constraint condition (3), random remove the goods inside, one goods at a time, determine whether to meet equation (3) after each removal, if it has met equation (3), the termination of the removal process; otherwise, then continue to random removal until equation (3) is satisfied.

An inadequate artificial fish coding is defined as a feasible artificial fish coding that does not make full use of all knapsacks, as well as the repair of infeasible artificial fish coding, random repair strategy is used. The random repair of the inadequate artificial fish coding refers to firstly the artificial fish coding is decoded, then looks for all goods not loaded in any knapsack, they are randomly put in the knapsacks of a remaining capacity, and equation (3) must be met when they are put in the knapsacks until all knapsacks can't been added goods.

The repair of the coding after foraging, clustering and tailgating and other behaviors. In this paper, an artificial fish coding is an integer coding, the coding after foraging are real number coding, so they are rounded down through the integral function, if the elements in the real number coding are not satisfied $a_{i j} \in[0, m+1)$, then assign them new random values, i.e. $a_{i j}=\operatorname{rand}() \in[0, m+1)$, and then the artificial fish coding after rounding down are repaired by the way mentioned in section 3.2, make them become adequate and feasible artificial fish coding, the coding after other behaviors also adopt the same method.

IAFSA steps for MKP. The process of IAFSA is shown in Fig.1.

\section{The experimental results and analysis}

To verify IAFSA for MKP, select 3 knapsacks, 10 goods of Multi-knapsack problem as an example, the capacities of knapsacks are 5.3, 4.5, 6.2 respectively, the weights and values of the goods are shown in table 2 , the problem of maximum value is 21.4 .

Table 2 The value and weight of goods

\begin{tabular}{ccccccccccc}
\hline Goods & 1 & 2 & 3 & 4 & 5 & 6 & 7 & 8 & 9 & 10 \\
\hline Value & 2.3 & 1.5 & 3.4 & 1.6 & 5.2 & 4.3 & 2.8 & 3.9 & 4.1 & 2.5 \\
Weight & 1.2 & 3.4 & 2.5 & 1.6 & 1.9 & 4.3 & 5.1 & 2.8 & 3.5 & 4.2 \\
\hline
\end{tabular}

Set $N=40$, visual $=4$, step $=2, \delta=0.618$, Try_number $=5$, the iteration number of each experiment is 9 , the experiment is repeated 20 times. To compare the performance of IAFSA, the greedy algorithm and the value density sorting method in [3] are applied to AFSA, hereinafter it is abbreviated as AFSA based on reference [3], the iteration numbers are 9 times and 25 times respectively, experimental results are shown in Table 3.

From table 3, IAFSA only need 0.52 seconds each experiment and the optimum solution can be obtained, the optimal solution can be obtained each time in 20 times experiments, each experiment takes no more than 9 times iteration and the convergence speed is fast. The optimal artificial fish coding is $[3,0,0,1,3,2,0,3,1,0]$, namely goods 4,9 are placed in knapsack 1 , goods 6 is placed in knapsack 2, goods 1, 5, 8 are placed in knapsack 3. As compared with AFSA based on reference [3], when its largest iterations number is nine times as same as IAFSA, only 12 times experiment can obtain the optimal solutions in 20 times experiments. When the maximum iterations number increases to 25 times, although the time of achieving the optimal solutions increases to 15 , the 
probability of the optimal solutions is only $75 \%$, there is still a big gap from $100 \%$ of IAFSA, and the average computation time is 3.16 seconds, it is six times as 0.52 seconds of IAFSA, Fig. 2 shows the average total knapsack value curves with the change of the iteration number.

Table 3 The comparison of 20 trials results

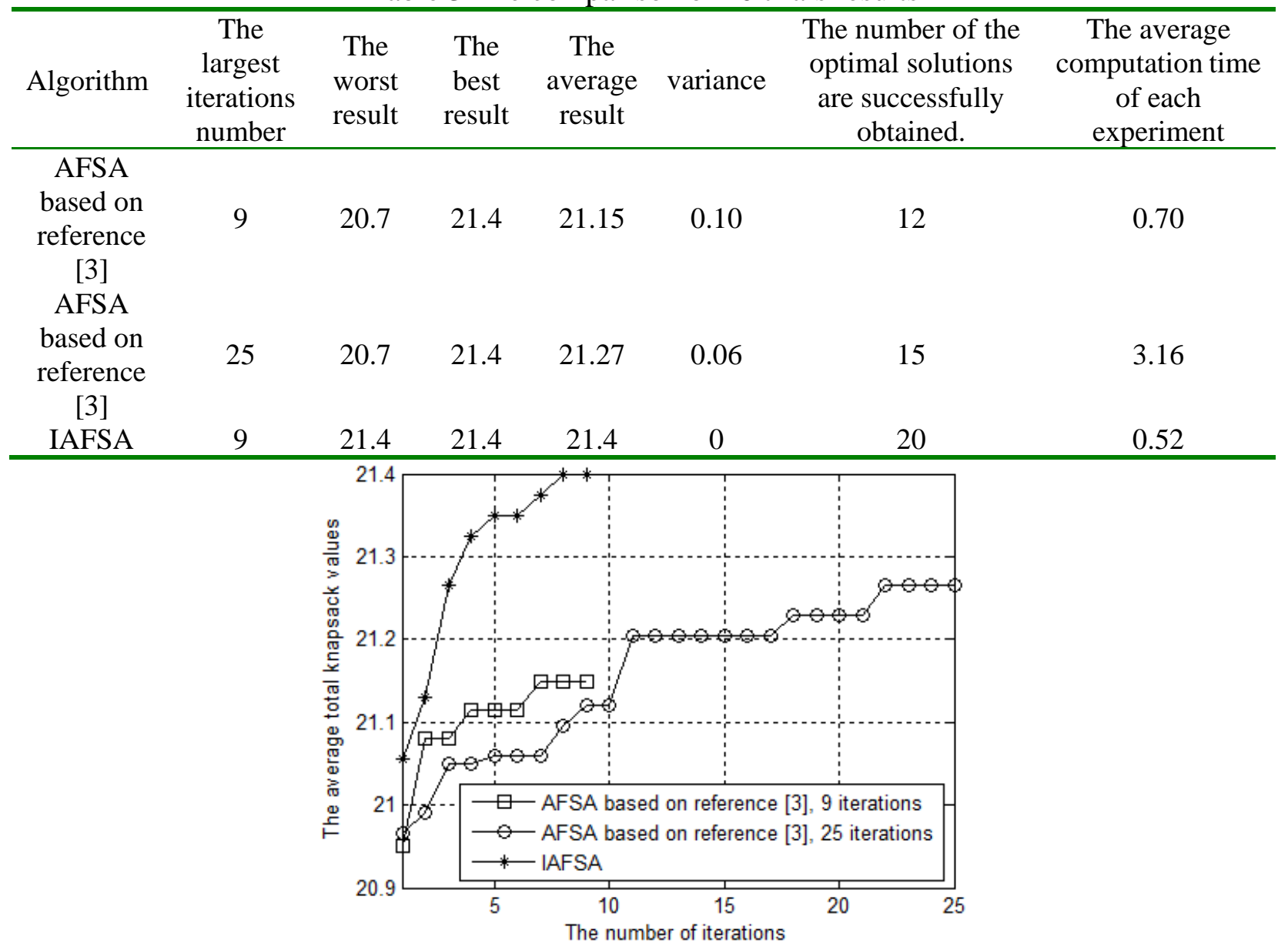

Fig. 2 The average total knapsack value curves with the change of the iteration number

It is shown in Fig.2 with the increase of the iteration number, IAFSA gradually approaches the maximum value of 21.4, and finally reaches the maximum value of 21.4, relative to AFSA based on the greedy algorithm and value density sorting strategy in reference [3], IAFSA has great advantage no matter on convergence speed, or convergence effect and operation time etc.

\section{Conclusion}

This paper presents an improved artificial fish school algorithm for MKP, the experimental results show that IAFSA has a good convergence and stability for small MKP, it can find the global optimal solution with $100 \%$ probability, how to apply it in large-scale MKP problem and better jump out of the trap of local optimal solutions will be the future research direction.

\section{Acknowledgements}

The work of this paper obtained the national natural science foundation of China (61179032/61072143), Science and technology plan projects of Hubei Province Education Department (B20091803/Q20131703), the authors also want to thank the anonymous reviews give constructive suggestions. 


\section{References}

[1] D. Pisinger, An exact algorithm for large multiple knapsack problems, European Journal of Operational Research, Vol. 114 (1999) No.3, p.528-541.

[2] A.B. Yu, J.B. Yang, Genetic algorithm for multi knapsack problem, Journal of Computing Technology and Automation, Vol. 21 (2002) No.2, p.59-63.

[3] H.S. Song, R.Y. Fu, R.S. Xu, et al, Hybrid genetic algorithm for multi-knapsack problem, Journal of Computer engineering and applications, Vol. 45 (2009) No.20, p.45-48.

[4] Z.H. Ren, J. Wang, 2009. A Discrete Particle Swarm Optimization for Solving Multiple Knapsack Problems. ICNC '09. Fifth International Conference on Natural Computation, Tianjin, China. IEEE, USA, p.166-170.

[5] W.Q. Xiong, P. Wei, B.C. Jiang, 2010. Binary ant Colony Algorithm with congestion control strategy for the 0/1 Multiple Knapsack problems. 8th World Congress on Intelligent Control and Automation (WCICA), Jinan, China. IEEE, USA. p.3296-3301.

[6] X.L. Li, Z.J. Shao, J.X. Qian, An optimizing method based on autonomous animates: fish-swarm algorithm, Systems Engineering Theory and Practice, Vol. 22 (2002) No. 11, p.32-38.

[7] X.L. Li, J.X. Q, Studies on artificial fish swarm optimization algorithm based on decomposition and coordination techniques, Journal of Circuits and Systems, Vol. 8 (2003) No. 1, p.1-6.

[8] X.L. Li, F. Lu, G.H. Tian, et al: Applications of Artificial fish school algorithm in Combinatorial optimization problems, Journal of Shandong University (Engineering Science), Vol. 34 (2004) No.5, p.64-67. 